\title{
ULTRA-HIGH FREQUENCY TRAIN OPERATION USING THE SOFT COUPLING TECHNIQUE: HEADWAY EVALUATIONS
}

\author{
FUMITAKA KAKUHAMA \& RYO TAKAGI \\ Kogakuin University, Japan
}

\begin{abstract}
The level of congestion observed in urban railways during peak hours is unacceptably high, and there is a strong need for quick improvement. One possible method to alleviate congestion without largescale investment in railway infrastructure (e.g. increasing tracks) is the realisation of "soft coupling". This is a concept in which the space between two moving trains is controlled to an extremely short distance so that these two trains look as if they were mechanically coupled. The purpose of this study is to evaluate the limits of the transport capacity of urban railways when soft coupling is applied. Although it is expected that ultra-high frequency train operation will be possible by using this technique, there is a danger that these two trains would collide with each other when doing so. To avoid the problem, deceleration parameters of trains must be set properly. In this paper, the authors first search for the appropriate set of deceleration parameters by means of simplified braking performance calculations. Following this, the authors evaluate the possibility of increasing train frequency by using this technique. Especially, the authors reveal that it is possible to reduce headways between two trains that are running in the same direction and calling at the same platform in a station even further by using soft coupling, when compared with the cases using either pure moving block (PMB), relative moving block (RMB), a new variant of continuous ATP used in Japan (called Digital ATC) and another conventional variant of continuous ATP used in Japan (called ATS). By comparing all five types of signalling systems, it can be concluded that soft coupling can greatly contribute to shortening the headways between trains.
\end{abstract}

Keywords: soft coupling, ultra-high frequency train operation, moving block, signalling system, the headways between the leading and the following trains.

\section{INTRODUCTION}

The level of congestion observed in the urban rail network during peak hours is unacceptably high, and there is a strong need for quick improvement. Meanwhile, innovative products and services, such as autonomous road vehicles and 5th generation mobile communication systems, are becoming available. The emergence of the autonomous road vehicles naturally means that, in the future, railways may lose the competitive edge in many parts of the transport market where railways dominate. At the same time, the emergence of these technologies will also mean there is a fair chance that railway services can be given a major facelift by utilizing them, opening the possibility for railways to survive in a new environment.

For railways, it would be most important to provide more transport capacity. Shortening the headways between trains would be effective in realising this, especially by introducing the advanced signalling and train control systems. Among such advanced systems is "soft coupling", and this research clarifies the limit of transport capacity when soft coupling is applied.

\section{TRAIN INTERVAL CONTROL [3]}

To operate multiple trains safely on a railway track, it is necessary to keep the distance between adjacent trains long enough to prevent collisions. 


\subsection{The "brick wall” assumption}

The "brick wall” assumption is a common concept of railway signalling in which trains are controlled so as not to collide with each other even when a leading train stops at the infinite deceleration. Although there is currently no signalling system in use on urban railways in revenue service without this safety concept, this assumption is generally considered as "too much on the safe side", and it is generally believed that train frequency can be increased by lifting this restriction. However, unlike road vehicles which collide with each other fairly often, it is believed that collision on railways would never be acceptable. Therefore, when considering a train control system that falls outside the safety concept of "brick wall" assumption, maximum deceleration and other parameters used for that system are to be properly set so collisions never take place.

\subsection{Fixed block signalling system}

In this system, the tracks are divided into sections called "blocks", which are in most cases several hundred meters long. For each block section, at maximum only one train is permitted to enter there. This simple principle prevents the collision of trains from happening. However, when the length of the block is long, the distance between trains will become longer than necessary, reducing frequency of train operation.

\subsection{Moving block signalling system}

In this system, the position until which a train that follows another is allowed to move will continuously change as the leading train moves. There are two variants of moving block signalling systems: one is the PMB (Pure Moving Block) that uses the position information of the leading train only, and the other is the RMB (Relative Moving Block) that will use the speed of the leading train in addition to its position. Under the moving block signalling system, the distance between trains will be shorter than the fixed block variants, except when the margin distance parameter for PMB and/or RMB is set to a longer value. However, since the distance between trains depends on the braking performance of the following train, it will become very long when the running speed of the following train becomes high.

\section{SOFT COUPLING}

Soft coupling is a concept of a novel train control system in which the distance between two adjacent running trains will be much shorter than the minimum distance of PMB or RMB, making them look as if they are mechanically coupled [1].

\subsection{The nature of timetabling: finding compromise among conflicting passengers' interests}

For any one railway passenger, it would be ideal if there were a train that would depart at the originating station without the waiting time, call at no intermediate station route, require no change and arrive at the destination station with the shortest travel time. This, of course, would be unrealistic for railways as a mass transit system; one should understand that the true needs of passengers are generally in conflict with each other, and that the planning of railway services inherently and inevitably include finding the compromise between these conflicting needs of different passengers.

The train timetable designed for a railway line will generally consist of a combination of fast and slow trains. Here, it should be noted that there is the certain upper limit of the number 
of trains per hour that can be planned on the route. This would mean that, if one want to increase the number of faster trains on a busy route, one might have to reduce the number of slower trains altogether, which would mean that passengers benefitting from the slower services may lose out while passengers using faster services enjoy more trains. If one can increase the number of trains on the route, then one can think of the better compromise between these users with conflicting interests.

Soft coupling will enable a drastic increase in train frequency. Unfortunately, it would then be very difficult to find the train paths for the simple-to-use "all-stopping" services on a railway route that makes full use of the features of ultra-high-frequency train operation that are to be made possible by soft coupling; the timetable would generally become way too complicated to do that. However, by introducing cautiously designed timetables, railway services that will better suit individual passengers' needs may be realised, by increasing the "classes" of services that the operators provide. This may be possible by increasing the number of trains and at the same time diversifying the selection of stations each train will be calling at. This will mean introducing a completely new set of stopping patterns that would never have been realized in the conventional train timetabling. By doing this, it is expected that the inconvenience of not being able to run all-stopping services are compensated, and passengers using smaller stations, as well as those using larger stations, can enjoy better services.

\subsection{The goal of train timetabling with soft coupling}

The new train operation model suitable for soft coupling will be explained hereunder. Take a fictitious railway line with six stations, namely Stations A through F, as an example. Let us assume there are two classes of trains in the conventional timetable, called the Express trains and the Local trains. The express trains will call at Stations A and F only, which are both large stations. Local trains call at all stations, and make connections with express trains at stations A and F. Table 1 shows the calculation conditions.

Fig. 1 shows the conventional train graph using the traditional "combined fast and slow" scheduling scheme. This is close to the upper limit train frequency, and because of that the express trains have to travel with longer-than-capable run time from Stations A to F because they catch up with the local trains before reaching $\mathrm{F}$ and have to slow down.

In the proposed timetabling concept incorporating soft coupling, which is illustrated in Fig. 2, each local train in Fig. 1 is divided into three shorter trains. Each of these shorter trains will now call at four out of six stations by adopting the skip-stop scheduling scheme [4] and

Table 1: Assumptions in the calculation.

\begin{tabular}{|l|c|c|c|c|}
\hline \hline \multirow{2}{*}{} & \multicolumn{2}{|c|}{ Conventional timetable } & \multicolumn{2}{c|}{ Proposed timetable } \\
\cline { 2 - 5 } (coaches/trainset) $\times$ (\# of trainsets) & $10 \times 1$ & $10 \times 1$ & $5 \times 2$ & $\begin{array}{c}3 \times 2 \\
4 \times 1\end{array}$ \\
\hline Dwell time (s) & 60 & 50 & 60 & 50 \\
\hline Interstation run time (s) & 60 & 60 & 60 & 60 \\
\hline Travel time between stations A and F (s) & 360 & 500 & 300 & 380 \\
\hline Line capacity (ratio) & \multicolumn{2}{|c|}{1} & & 2 \\
\hline Stations to be called at & A, F & A,B,C,D,E,F & A, B, F & 4 out of 6 \\
\hline
\end{tabular}




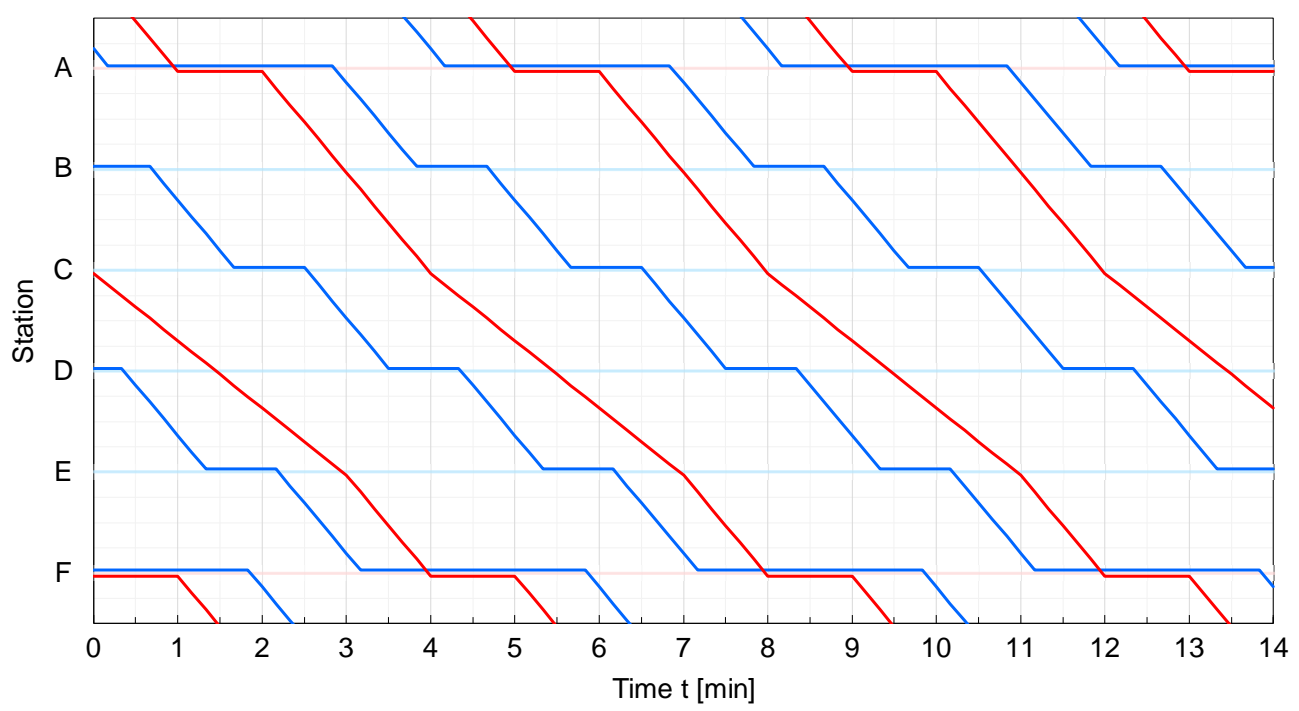

Figure 1: An example train graph using conventional scheduling scheme.

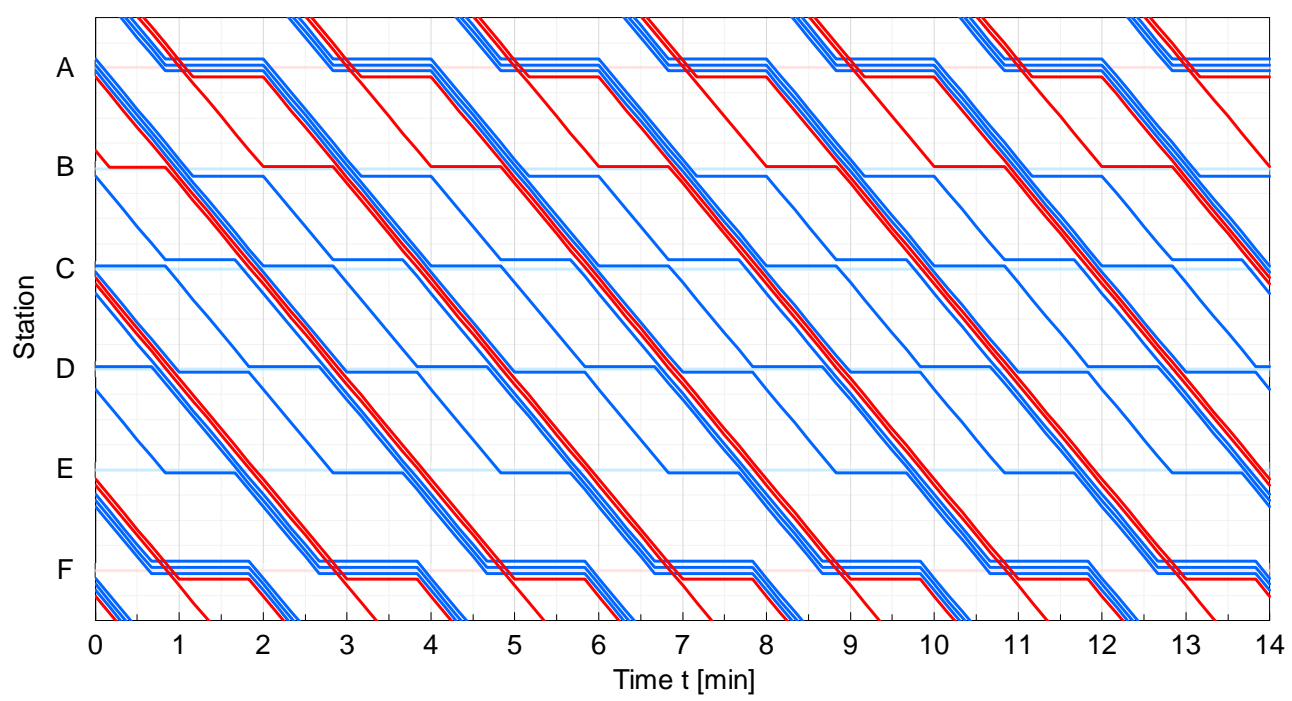

Figure 2: An example concept using proposed scheme incorporating soft coupling.

will run slightly faster than the all-stopping local trains in Fig. 1. This makes the express trains free from catching up with local trains, and they can now run faster than in Fig. 1. It can also be noted that the frequency of trains as a whole, and hence the transport capacity of the route, will also become significantly higher in Fig. 2 than in Fig. 1.

It should be noted, however, that what Fig. 2 shows is only a design concept at this stage. A more detailed timetable design should be carried out, based on the analyses on dwell times and headways. The intention of this paper is to give the basis for such analyses. 


\subsection{The method of control for soft coupling}

In soft coupling, the distance between trains is shorter than any existing signalling systems. It means that the extremely fast and precision control is required for soft coupling. There are five kinds of information necessary to control a train when it is soft-coupled with another in front or in rear. It requires the current value and the control target value of the velocity and acceleration. Furthermore, the relative distance between trains is necessary.

In the currently available variants of PMB systems, generally there is an error of about $0.5 \%$ in the train position measurement incorporated in them, which would be 0.5 meter error per 100 meters. In soft coupling, the distance between trains is 0.5 meters at the shortest. Although the relative speed is small, there is a danger of collision with trains if the error of train position detection is too large. Therefore, it is impossible to apply the train position measurement subsystems for the current PMB systems as they are to soft coupling.

Also, generally the information would be transmitted through the wireless inter-train communication link. The communication link should have very short communication cycles and minimal delays, and should be very reliable. Furthermore, during the soft-coupled operation, trains keep running even while the wireless communication is interrupted temporarily. It is never acceptable for two trains to collide even under any conditions, so a number of backup techniques should be found [2].

Fortunately, recent years have seen the emergence of many techniques that will help realise soft coupling. For example, Cooperative Adaptive Cruise Control (CACC) system, which control between vehicles distance with high accuracy, is already in practical use in road vehicles. Also, the next-generation mobile communication system (5G), with a communication delay of 1 millisecond or less, is to be introduced in the near future.

\section{HEADWAY EVALUATIONS}

The authors compared soft coupling and other four signalling systems - a conventional continuous ATP (ATS), a newer continuous ATP (Digital ATC: D-ATC), PMB and RMB for comparison to show that soft coupling contributes to shorter headways.

\subsection{Assumed conditions}

It is assumed that a leading train, dwelling at a platform of a station, departs at time $t=0$, and a following train will arrive at the same platform, and the departure-to-arrival headway will be calculated for different signalling systems.

It is also assumed that the following train approaches the station with the constant cruising speed of $80 \mathrm{~km} / \mathrm{h}$ before any needs for reducing speed arise when coming very close to the platform and/or the leading train.

In principle, under any signalling system, trains always try to accelerate at maximum acceleration with infinite jerk unless they either have reached the predefined cruising speed or need to apply brake to come to a halt at the platform. If the train exceeded the speed permitted by the signalling system, the train would decelerate using the (strongest) service brake. For fixed block variants, namely ATS and D-ATC, because the limit of movement authority changes step by step (not continuously as in the case of PMB and RMB) whenever the tail of the leading train moves out of a block section, the train does repeat maximum acceleration and deceleration. For PMB, RMB and soft coupling, a train that follows another with the minimum separation as permitted by the signalling system by which the track is controlled tries to keep the separation to be minimal as long as it can, which will result in acceleration or deceleration below maximum. 
Parameters assumed in this study are shown in Tables 2 and 3.

To make calculations easier, the following assumptions were made: The acceleration and deceleration parameters were assumed to be constant; the delays in the response of the driving operations (acceleration and braking) or in communication of information were assumed as zero; and the jerk (the rate of change in acceleration) was assumed as infinite. To compensate this, the acceleration and deceleration parameters assumed are slightly lower than the most typical values in Tokyo.

\subsection{Train trajectories}

The example train trajectories calculated based on Tables 2 and 3 are shown in Figs 3 and 4. As shown in Fig. 3, it is assumed that the length of the trains are 200 metres, and the platform spans between positions $x=1,000 \mathrm{~m}$ and $x=1200 \mathrm{~m}$. For the fixed block systems, namely ATS and D-ATC, the positions of block sections are also shown in Fig. 3.

Table 2: Assumed train length, maximum velocity, and information update cycle.

\begin{tabular}{|c|c|c|c|}
\hline \multirow{2}{*}{ Length L (m) } & \multicolumn{2}{|c|}{ Maximum velocity V (km/h) } & \multirow{2}{*}{$\begin{array}{l}\text { Update cycle of information } \\
\qquad \mathrm{T}(\mathrm{ms})^{\dagger 1}\end{array}$} \\
\hline & Leading train & Following train & \\
\hline 200.0 & \multicolumn{2}{|c|}{80.0} & 20 \\
\hline
\end{tabular}

$\dagger 1$ : Position, speed and acceleration of a train will be calculated based on information of the previous update cycle.

Table 3: Assumed acceleration and deceleration parameters and equations.

\begin{tabular}{|c|c|c|c|c|c|c|}
\hline & \multirow{2}{*}{$\begin{array}{l}\text { Leading } \\
\text { train }\end{array}$} & \multicolumn{4}{|c|}{ Following train } \\
\hline & & & Fixed block & PMB & RMB & Soft coupling \\
\hline \multicolumn{2}{|c|}{$\begin{array}{l}\text { Acceleration } \\
\alpha(\mathrm{km} / \mathrm{h} / \mathrm{s})\end{array}$} & 2.50 & $2.50^{\dagger 1}$ & $1.74^{\dagger 1}$ & $2.32^{\dagger 1}$ & $2.50^{\dagger 1}$ \\
\hline \multicolumn{2}{|c|}{$\begin{array}{l}\text { Acceleration/ } \\
\text { deceleration } \\
\beta_{B}(t)(\mathrm{km} / \mathrm{h} / \mathrm{s})\end{array}$} & - & - & \multicolumn{2}{|c|}{$\beta_{N} \times\left\{1-\left(\frac{\beta_{U}-\beta_{N}}{\beta_{U}}\right)\right.$} & $\left.\frac{v_{A}(t-\Delta t)}{v_{B}(t-\Delta t)}\right\}$ \\
\hline \multirow{3}{*}{ 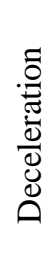 } & $\begin{array}{l}\text { Normal } \beta_{N} \\
(\mathrm{~km} / \mathrm{h} / \mathrm{s})\end{array}$ & 3.00 & 3.00 & 3.00 & 3.00 & 3.00 \\
\hline & $\begin{array}{l}\text { Emergency } \\
\beta_{E}(\mathrm{~km} / \mathrm{h} / \mathrm{s})^{\dagger 2}\end{array}$ & 4.00 & 4.00 & 4.00 & 4.00 & 4.00 \\
\hline & $\begin{array}{l}\text { Unusual } \beta_{U} \\
(\mathrm{~km} / \mathrm{h} / \mathrm{s})\end{array}$ & - & - & $\infty$ (Infinity) & 5.40 & - \\
\hline \multicolumn{2}{|c|}{ Margin distance $^{\dagger 3}$} & - & 20 & 20 & 20 & 0.5 \\
\hline
\end{tabular}

$\dagger 1$ : Under PMB, RMB or soft coupling, when there are a leading train that has come to a halt and a following train that has also come to a halt just behind the leading train, they will restart simultaneously as the leading train decides to start. Under fixed block systems, the following train restarts immediately after the next block section becomes unoccupied.

$\dagger 2$ : The calculations are based on normal deceleration parameters; emergency deceleration parameters are for reference only.

†3: For PMB, RMB and soft coupling, minimal distance between head of the following train and tail of the leading train. For fixed block systems, minimal distance between the head of the following train and the end of the block section closest to the one occupied by the leading train. 


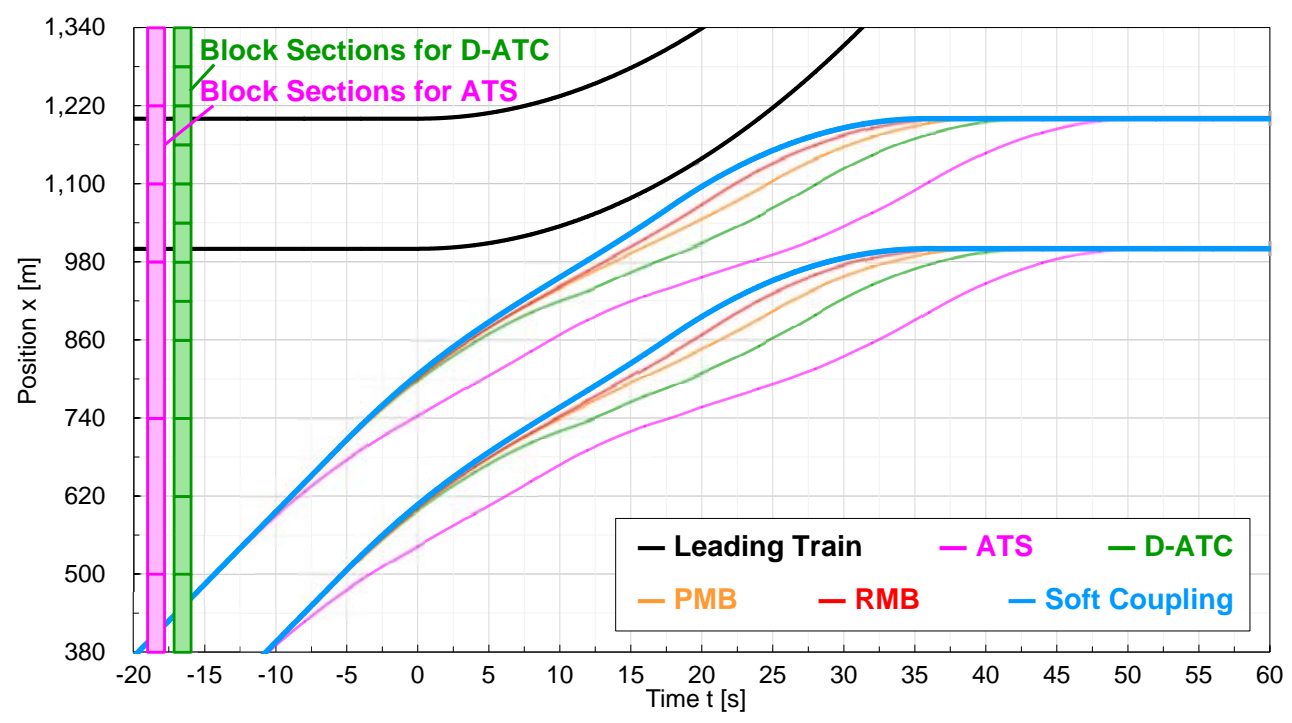

Figure 3: Comparison of trajectories of the nose and tail positions of trains (Case 2 in Table 4).

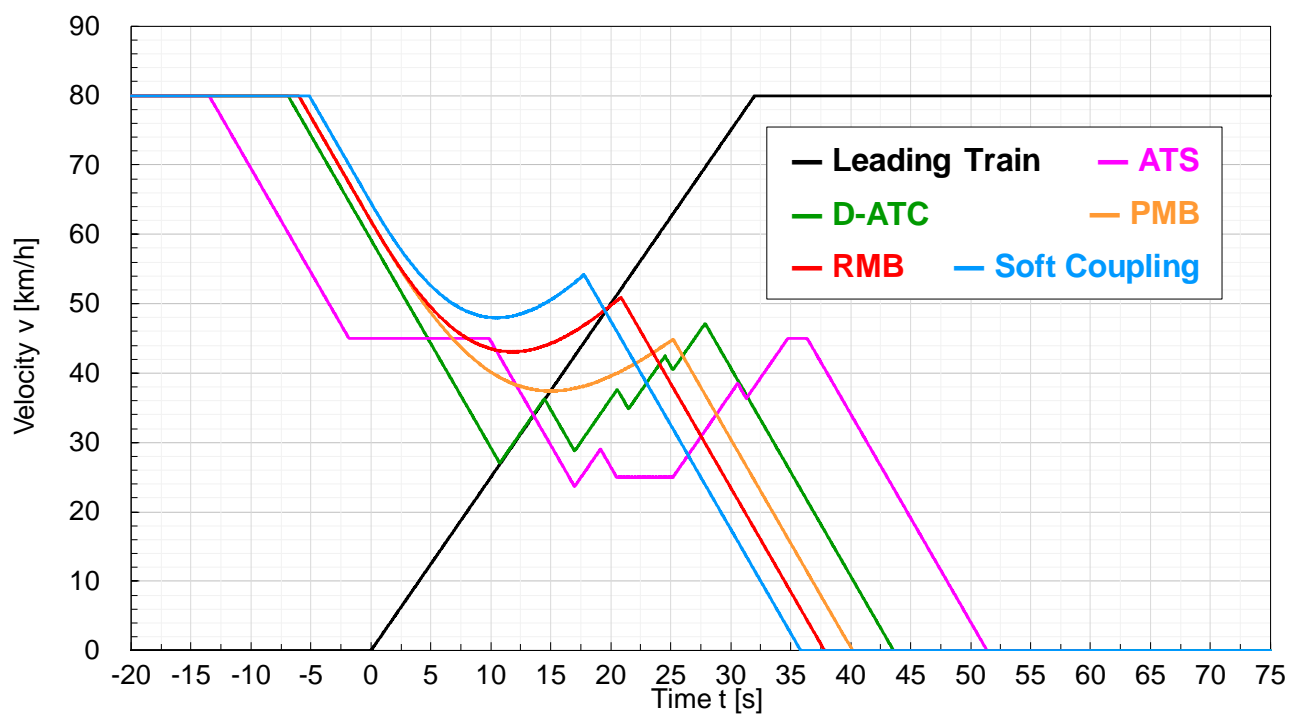

Figure 4: Comparison of velocity-time curves (Case 2 in Table 4).

The authors roughly classified the situations into three scenarios. The first is when the movement of the following train is affected by the leading train in the way that the following train is forced to come to a halt and re-accelerate before entering the stopping position on the platform in the destination station. The second is when the movement of the following train 
Table 4: Comparison of calculated departure-to-arrival headways.

\begin{tabular}{|c|c|c|c|c|c|c|}
\hline \multicolumn{2}{|c|}{$\begin{array}{l}\text { Behavior of the following train due to } \\
\text { approach to the leading train }\end{array}$} & $\begin{array}{l}\text { ATS } \\
(\mathrm{s})\end{array}$ & $\begin{array}{l}\text { D-ATC } \\
(\mathrm{s})\end{array}$ & $\begin{array}{l}\text { PMB } \\
(\mathrm{s})\end{array}$ & $\begin{array}{c}\mathrm{RMB} \\
(\mathrm{s})\end{array}$ & $\begin{array}{l}\text { Soft coupling } \\
\text { (s) }\end{array}$ \\
\hline \multicolumn{2}{|c|}{$\begin{array}{l}\text { Extra halt before entering the } \\
\text { platform }\end{array}$} & 52.7 & 46.4 & 38.8 & 35.7 & 32.6 \\
\hline Severe extra deceleration & Case 1 & 52.7 & 46.4 & 39.0 & 35.9 & 33.1 \\
\hline $\begin{array}{l}\text { Moderate extra } \\
\text { deceleration }\end{array}$ & Case 2 & 51.4 & 43.6 & 40.2 & 37.9 & 35.8 \\
\hline Slight extra deceleration & Case 3 & 54.2 & 45.9 & 43.9 & 42.5 & 41.5 \\
\hline \multicolumn{2}{|l|}{ No extra deceleration } & 69.3 & 54.6 & 51.9 & 47.6 & 44.5 \\
\hline
\end{tabular}

is affected by the leading train in the way that the following train is only forced to decelerate and re-accelerate without come to a halt before entering the station. The last is when the movement of the following train is not affected by the leading train. The calculated departureto-arrival headways in each case are shown in Table 4. In Table 4, the figure in red bold face under each signalling system indicates that it is the shortest calculated departure-to-arrival headway for the signalling system.

Here, the second scenario was subdivided into three cases. Different approach time of the following train is assumed for each case. The approach time is the time the following train passes a certain point behind the platform at the constant cruising speed. The approach time for Case 3 was determined so that the following train has to decelerate under any signalling systems in the table. The approach times for Cases 2 and 1 were determined by subtracting 10 seconds and 20 seconds from that of Case 3, respectively, meaning that the deceleration will be severer. The same approach times are used for all signalling systems.

Note that for the results in Fig. 4 of the conventional fixed block variants of the signalling systems, namely ATS and D-ATC, because the limit of movement authority changes step by step (not continuously as in the case of PMB, RMB and Soft coupling) whenever the tail of the leading train moves out of a block section, the following train does repeat maximum acceleration and deceleration, as has already been explained in Section 4.1.

These results clearly show that the headways are different depending on the signalling system. For fixed block signalling systems, it is known that the departure-to-arrival headway when the following train comes to a complete halt behind the train dwelling at the platform will be longer than the headway when the following train decelerates to some extent but does not completely come to a halt. In contrast, one of the authors, Takagi, has revealed that, under the PMB, the shortest departure-to-arrival headway at a platform will be yielded when the following train comes to a complete halt behind the preceding train dwelling at the platform [5]. The results correspond very well with these previous findings by the authors.

The results presented in Table 4 show the fact that the departure-to-arrival headway under $\mathrm{PMB}, \mathrm{RMB}$ or soft coupling is minimised when the following train comes to a complete halt. This contradicts the common knowledge that the train that follows another should NOT come to a halt before entering the platform in order to achieve minimal headways. The authors believe that this common knowledge is actually based on calculations for the fixed block signalling systems, under which the limit of movement authority changes step by step, and should not be applied to moving block signalling systems and more advanced systems such 
as soft coupling, under which the limit of movement authority moves continuously according to the position of the leading train.

However, it should be noted that, if the following train approached the leading train dwelling at the platform earlier, the following train would be forced to decelerate or stop before entering the platform, and therefore the start-to-stop time from the previous station to the platform in question will increase. As a future work, the evaluation of start-to-stop time together with the headways must be done.

\section{CONCLUSIONS}

The authors revealed that soft coupling is effective in shortening the headways. It contributes to further few seconds of shortened headways compared with the PMB signalling system.

However, many would consider that the need to move away from the traditional safety concept of "brick wall" assumption would make soft coupling unacceptable. It would therefore be necessary to find a workaround so that headways shorter than the currently available PMB can be realised under the "brick wall” assumption.

\section{ACKNOWLEDGEMENT}

This work has been supported by JSPS KAKENHI Grant Number JP 16 K06236.

\section{REFERENCES}

[1] Moriya, O. \& Sone, S., Soft-coupling. J. SICE, 32(7), pp. 600-603, 1993. (In Japanese.)

[2] Kakuhama, F. \& Takagi, R., Possibility of ultra-high frequency train operation. 24th Joint Symposium on Railway Technology (J-Rail 2017), S9-4, 2017. (In Japanese.)

[3] Takeuchi, H., Goodman, C.J. \& Sone, S., Moving block signalling dynamics: performance measures and re-starting queued electric trains. IEE Proc. Electr. Power Appl., 150(4), pp. 483-492, 2003.

[4] Shimizu, T. \& Takagi, R., Ultra-high frequency train operation for the realization of Ultra-Convenient Rail Transport (UCRT), Rail Lille 2017 Conference, Lille, France, 2017.

[5] Takagi, R., Synchronisation control of trains on the railway track controlled by the moving block signalling system. IET Electr. Syst. Transp., 2(3), pp. 130-138, 2012. 